\title{
Expression profiles of miRNAs in Gossypium raimondii"
}

\author{
Jun $\mathrm{MA}^{1}$, Teng-long GUO ${ }^{2}$, Qing-lian $\mathrm{WANG}^{3}$, Kun-bo $\mathrm{WANG}^{4}$, Run-run $\mathrm{SUN}^{1,3}$, Bao-hong ZHANG ${ }^{\dagger 1,3}$ \\ $\left({ }^{I}\right.$ Department of Biology, East Carolina University, Greenville, NC 27858, USA) \\ $\left({ }^{2}\right.$ Heze Academy of Agricultural Sciences, Heze 274000, China $)$ \\ $\left({ }^{3}\right.$ Henan Collaborative Innovation Center of Modern Biological Breeding, Henan Institute of Science and Technology, Xinxiang 453003, China) \\ $\left({ }^{4}\right.$ Cotton Research Institute, Chinese Academy of Agricultural Sciences, Anyang 455000, China) \\ †E-mail: zhangb@ecu.edu
}

Received Oct. 18, 2014; Revision accepted Jan. 31, 2015; Crosschecked Mar. 18, 2015

\begin{abstract}
RNAs are a class of conserved, small, endogenous, and non-protein-coding RNA molecules with 20-24 nucleotides (nt) in length that function as post-transcriptional modulators of gene expression in eukaryotic cells. Functional studies have demonstrated that plant miRNAs are involved in the regulation of a wide range of plant developmental processes. To date, however, no research has been carried out to study the expression profiles of miRNAs in Gossypium raimondii, a model cotton species. We selected 16 miRNAs to profile their tissue-specific expression patterns in G. raimondii four different tissues, and these miRNAs are reported to play important roles in plant growth and development. Our results showed that the expression levels of these miRNAs varied significantly from one to another in a tissue-dependent manner. Eight miRNAs, including miR-159, miR-162, miR-164, miR-172, miR-390, miR-395, miR-397, and miR-398, exhibited exclusively high expression levels in flower buds, suggesting that these miRNAs may play significant roles in floral development. The expression level of miR-164 was relatively high in shoots beside flower buds, implying that the function of miR-164 is not only limited to floral development but it may also play an important role in shoot development. Certain miRNAs such as miR-166 and miR-160 were extremely highly expressed in all of the four tissues tested compared with other miRNAs investigated, suggesting that they may play regulatory roles at multiple development stages. This study will contribute to future studies on the functional characterization of miRNAs in cotton.
\end{abstract}

Key words: Cotton, miRNA, Expression profiles, Quantitative real-time PCR (qRT-PCR), Gossypium raimondii doi: 10.1631 jzus.B1400277

Document code: A

CLC number: S562

\section{Introduction}

miRNAs are a class of well conserved, small, endogenous, and non-protein-coding RNA molecules with 20-24 nucleotides (nt) in length that function as post-transcriptional modulators of gene expression in eukaryotic cells through cleavage of target mRNAs or translational inhibition (Carrington and Ambros,

\footnotetext{
¿ Corresponding author

* Project supported by the US National Institute of Food and Agriculture (NIFA), the Cotton Incorporated, and the National Natural Science Foundation of China (No. 31170263)

(DD ORCID: Jun MA, http://orcid.org/0000-0003-4354-2523

(C) Zhejiang University and Springer-Verlag Berlin Heidelberg 2015
}

2003; Bartel, 2004; Millar and Waterhouse, 2005; Zhang et al., 2007). Mature miRNAs are derived from miRNA coding genes with their lengths varying widely from 60 to >1000 nt (Bartel, 2004; Kurihara and Watanabe, 2004; Lee et al., 2004). Initially encoded by miRNA genes with RNA polymerase (RNApol) II or RNApol III, primary miRNAs (pri-miRNAs) are further processed by the RNase III enzyme Drosha in animals or Dicer-like 1 (DCL1) in plants to form single-stranded stem-loop intermediates known as pre-miRNAs, which are then cleaved into miRNA:miRNA* duplexes in plants (Bartel, 2004; Kurihara and Watanabe, 2004; Lee et al., 2004). Methylated by Hua Enhancer 1, the miRNA 
duplexes are further transported by HASTY in plants into cytoplasm where the mature miRNAs enter the RNA-induced silencing complex (RISC) containing Argonaute 1 (AGO1) to regulate gene expression, whereas the miRNA*s are degraded (Papp et al., 2003; Bartel, 2004; Park et al., 2005). As miRNAs are perfectly or near-perfectly complementary to their target sequences in plants, instead of translational inhibition, the cleavage of target miRNAs becomes the predominant regulation mechanism of plant miRNAs.

Currently, both computational and experimental approaches have been employed to identify miRNAs and miRNA targets, as well as their specific functions. Up to now, there are 28645 miRNA entries in the miRNA available public database miRBase (Release 21, June 2014). Functional studies have demonstrated that plant miRNAs are involved in the regulation of a wide range of plant developmental processes, such as plant organ development, response to environmental stress, signal transduction, and disease resistance (Zhang et al., 2006; Zhang and Wang, 2015). For instance, miR-172, which targets a class of APETALA2 (AP2)-like genes involved in plant flowering and floral organ identity, plays a crucial role in floral development as well as the phase transition from vegetative growth to reproductive growth, whereas miR-395 was shown to be induced by abiotic stresses such as drought and sulphate deprivation, indicating its role in plant response to severe environmental conditions (Zhang et al., 2006).

Cotton is thought to be one of the most important fiber-producing and economic crops around the world, considering that fiber products and cooking oil made out of cotton have become indispensable parts of our daily lives. It was reported that cotton has been grown in more than 100 countries, accounting for $2.5 \%$ of the world's arable land. Current estimates for the total international trade are about 133 billion USD annually, including raw cotton as well as relevant products and services, which further create about 350 million jobs worldwide (Ma et al., 2014). It has been found that cotton miRNAs may perform a wide variety of functions, such as ovule, fiber, leaf, and flower development, which makes it important to obtain a better understanding of cotton miRNAs in order to improve cotton yield.
Compared with other plant species, miRNArelated research in cotton has not progressed very far. Although there are several reports on cotton miRNA identification using both computational and experimental approaches, only a few research projects have been carried out to study the expression profiles of miRNAs in cotton. One of the major reasons is that cotton is a tetraploid (AADD) species with a complicated genome. Recently, Gossypium raimondii, a wild Central American cotton species, has been sequenced and has become a model cotton species. Currently, the genome-wide survey of cotton small RNAs has been conducted using deep sequencing technology. The survey is concerned with the composition and expression of conserved miRNA genes in diploid cotton. It investigated the evolution of 33 conserved miRNA genes in $G$. arboreum and $G$. raimondii, and compared the expressions of these miRNA genes and miRNA-targeted genes between the two diploid cotton species (Gong et al., 2013). Studies have also revealed that miRNAs may be involved in cotton fiber initiation and development (Xue et al., 2013; Liu et al., 2014; Xie et al., 2015). However, no systemical study has been performed on G. raimondii miRNAs in terms of expression profile in different tissues, which limits the application of miRNA biology on cotton improvement as well as basic genetics studies. In order to gain more insights into the tissue-specific expression profiles of miRNAs in G. raimondii, we selected 16 miRNAs to profile their expression profiles in four different $G$. raimondii tissues. The 16 miRNAs were miR-156, miR-159, miR-160, miR-162, miR-164, miR-166, miR-167, miR-169, miR-172, miR-390, miR-393, miR-395, miR-396, miR-397, miR-398, and miR-399, which are reported to play important roles in the growth and development of rice as well as Arabidopsis. Our results showed that the expression levels of these miRNAs varied significantly from one to another, indicating some degrees of tissue specificity.

\section{Materials and methods}

\subsection{Plant materials}

G. raimondii seeds were planted in the greenhouse of East Carolina University (USA) at $24-27^{\circ} \mathrm{C}$ using regular agronomic practices. During the flowering 
season, plant tissues of leaves, flower buds, shoots, and sepals were harvested separately and wrapped in aluminum foil. After immediately freezing in liquid nitrogen, all collected cotton tissues were stored at $-80{ }^{\circ} \mathrm{C}$.

\subsection{RNA isolation}

Total RNAs were isolated from cotton leaves, flower buds, shoots, and sepals using the mirVana ${ }^{\mathrm{TM}}$ miRNA Isolation Kit (Ambion, Austin, TX, USA) according to the manufacturer's instructions. In brief, after being put into a 2-ml centrifuge tube with Lysis/ Binding Buffer, the ground cotton tissues were homogenized for 15-20 s using an Ultrasonic Convertor on ice. Subsequently, the tissue lysate was mixed with miRNA Homogenate Additive by vortexing. After being left in the ice for $10 \mathrm{~min}$, the tissue lysate was thoroughly mixed with acid-phenol:chloroform and further centrifuged at maximum speed $(10000 \mathrm{~g})$ at room temperature to separate the organic phase and the aqueous phase, which was then transferred to a new microcentrifuge tube with $100 \%$ ethanol. Collected with a filter cartridge, the total RNAs were then purified by wash solutions. Finally, the total RNAs were recovered by adding nuclease-free water to the filter. The total RNA quantity and purity were assessed by a NanoDrop ND-1000 Spectrophotometer (NanoDrop Technologies, Wilmington, DE, USA).

\subsection{Real-time quantitative reverse transcription polymerase chain reaction (RT-PCR) analysis}

The single-stranded complementary DNA (cDNA) was synthesized from $1 \mu \mathrm{g}$ of total RNAs using the TaqMan $^{\circledR}$ MicroRNA Reverse Transcription Kit (Applied Biosystems, Foster City, CA, USA) and a miRNA specific reverse transcription (RT) primer for each miRNA. A total of 16 miRNAs were analyzed, which included miR-156, miR-159, miR-160, miR-162, miR-164, miR-166, miR-167, miR-169, miR-172, miR-390, miR-393, miR-395, miR-396, miR-397, miR-398, and miR-399. The reason for selected these miRNAs was that they were reported to play important roles in the growth and development of various plant species, including Arabidopsis and rice. The real-time RT-PCR was subsequently carried out with a 7300 Applied Biosystems Real-Time PCR System. The components of each reaction mixture were as follows: $2 \mu 1$ of DNase/RNase-free water, $5 \mu 1$
Real-Time SYBR Green PCR Master Mix, $1 \mu 1$ diluted cDNA product from RT-PCR reaction, and $2 \mu \mathrm{l}$ gene-specific miRNA primers. There were three biological replicates for each cotton organ and each biological replicate was run three times to minimize the chance of error. The temperature program included enzyme activation at $95{ }^{\circ} \mathrm{C}$ for $10 \mathrm{~min}$, denature at $95{ }^{\circ} \mathrm{C}$ for $15 \mathrm{~s}$, and annealing/elongation at $60{ }^{\circ} \mathrm{C}$ for $60 \mathrm{~s}$. Two reference genes, SAD1 and $T U A 11$, were used to normalize the expression values. The equation $R=2^{-\left(C_{11}-C_{12}\right)}$ was used to calculate the relative expression levels $(R)$, in which $C_{\mathrm{t} 1}$ is the $C_{\mathrm{t}}$ value of the $T C P$ genes, while $C_{\mathrm{t} 2}$ represents the $C_{\mathrm{t}}$ value of the reference gene. Subsequently, the software MultiExperiment Viewer $(\mathrm{MeV})$ was employed to generate a heat map for gene expression patterns.

\section{Results}

Except for miR-399, all of the 16 tested miRNAs were expressed in the four different cotton tissues (Figs. 1 and 2). The expression levels of those expressed miRNAs varied significantly from one to another in a tissue-dependent manner.

Many tested miRNAs exhibited exclusively high expression levels in a specific tissue (Figs. 1 and 2). For instance, the expression levels of eight miRNAs, including miR-159, miR-162, miR-164, miR-172, miR-390, miR-395, miR-397, and miR-398, were highly expressed in the flower buds, while miR-156 was expressed at much higher levels in leaves compared with other tissues tested, implying that they may play important regulatory roles in the corresponding tissues. In addition, some miRNAs were observed to be expressed preferentially in two tissues. For example, miR-396 displayed especially high expression levels in flower buds and sepals, whereas miR-160 was highly expressed in flower buds and shoots. Moreover, it is also remarkable that a few miRNAs were constitutively expressed in all tissues tested at either very high or very low levels. miR-166, for instance, showed continually high expressions in all the four tissues, suggesting that it may play regulatory roles at multiple development stages in cotton, whereas the expression levels of miR-169 were much lower in every organ tested than that of most other miRNAs by tens- to thousands-fold (Figs. 1 and 2). 


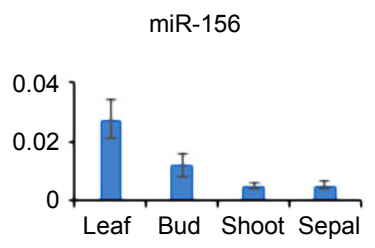

miR-162

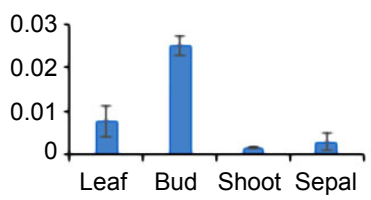

miR-167
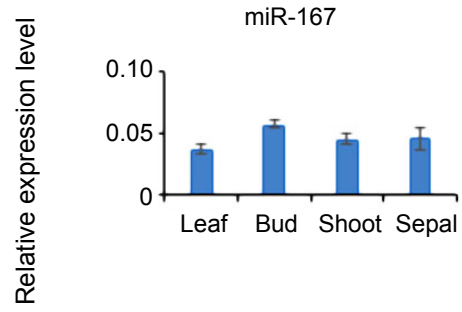

miR-390

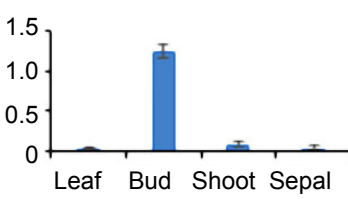

miR-396

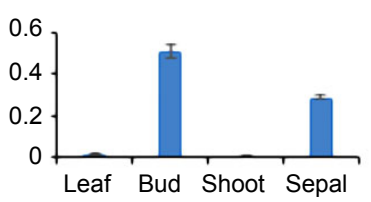

miR-159

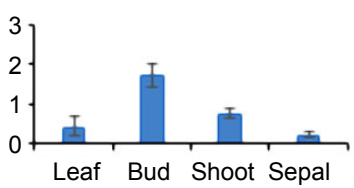

miR-164

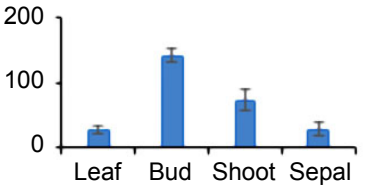

miR-169

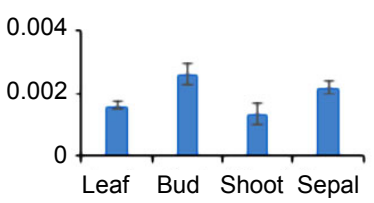

miR-393

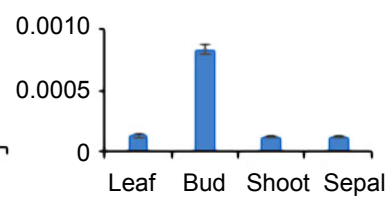

miR-397

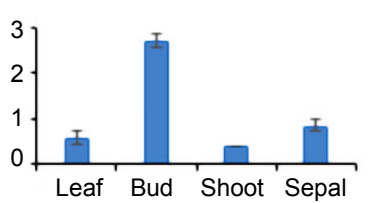

miR-160

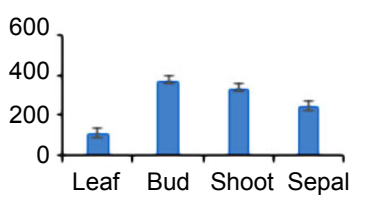

miR-166

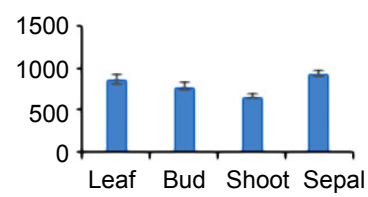

miR-172

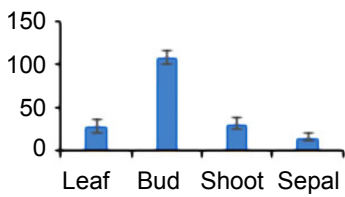

miR-395

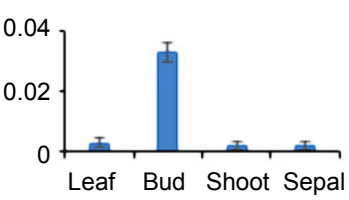

miR-398

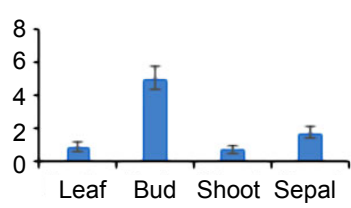

Fig. 1 Expression profiles of 15 miRNAs across different tissues

The $y$-axis represents the relative expression levels of miRNAs against reference genes. Error bars indicate standard deviations for three replicates. We did not include miR-399 because it was not expressed in all tested four cotton tissues

In the cotton leaf, miR-166 displayed the highest expression level among the 16 miRNAs detected, followed by miR-160 (Figs. 1 and 2). The expression levels of miR-166 and miR-160 were 1150 -fold and 121 -fold of the mean value of the 15 miRNAs, respectively. In contrast, miR-393, miR-169, and miR-162 exhibited relatively low expression levels compared with the other miRNAs tested. For example, the expression level of miR-162 was only less than 0.001 -fold of the average expression levels of the 15 miRNAs. In addition to the cotton leaf, miR-166 and
miR-160 also showed similar expression profiles in flower buds, shoots and sepals. The expression levels of miR-166 and miR-160 were relatively high compared with other miRNAs tested in these three tissues. Similarly, miRNAs, such as miR-393, miR-169, and miR-162, which are lowly expressed in cotton leaves, also displayed relatively low expression levels in flower buds, shoots, and sepals. Additionally, it is noticeable that although certain miRNAs were expressed preferentially in one or two tissues, it does not necessarily mean that they have the highest expression 
levels in the corresponding tissues among all miRNAs examined. miR-156, for example, is the only miRNA that showed exclusively high expression level in leaves according to the qRT-PCR results. However, among all of the 16 miRNAs, it is miR-166 that exhibited the highest expression level in cotton leaves. A similar circumstance was also observed in the shoots. Such circumstances were mainly caused by the fact that certain miRNAs such as miR-166 and miR-160 were extremely highly expressed in all the four tissues tested.

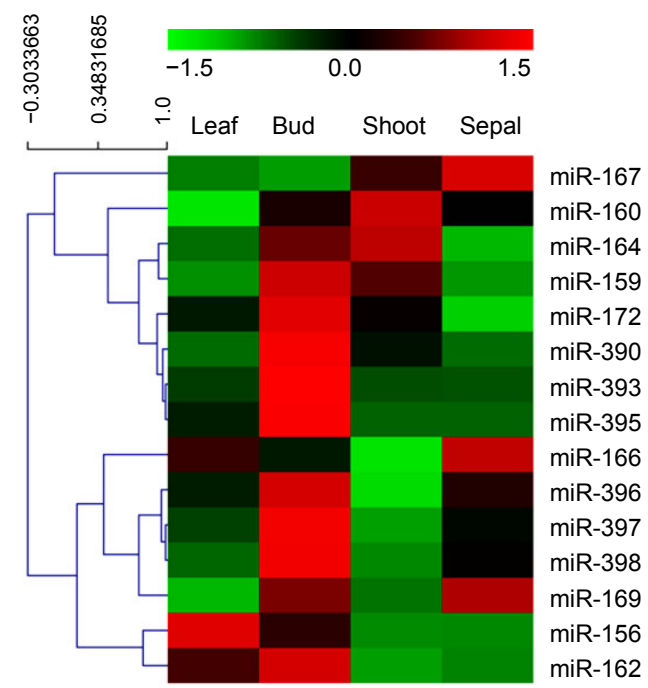

Fig. 2 Heat map representation for expression patterns of 15 miRNAs across different tissues

The expression profile data of 15 miRNAs in leaves, buds, shoots, and sepals were obtained through quantitative realtime PCR. The equation $R=2^{-\left(C_{11}-C_{12}\right)}$ was used to calculate the relative expression levels $(R)$, in which $C_{\mathrm{t} 1}$ is the $C_{\mathrm{t}}$ value of $T C P$ genes, while $C_{\mathrm{t} 2}$ represents the $C_{\mathrm{t}}$ value of the reference gene. The software MultiExperiment Viewer $(\mathrm{MeV})$ was employed to generate the heat map for gene expression patterns. We did not include miR-399 because it was not expressed in all tested four cotton tissues

\section{Discussion}

It has been widely reported that miRNAs participate in the regulation of multiple aspects of plant growth and development, such as leaf development, floral development, shoot, root, and fiber development, response to environmental stress, and signal transduction (Carrington and Ambros, 2003; Millar and Waterhouse, 2005; Zhang et al., 2007). In a recent study, for example, 33 conserved miRNA gene families were identified in G. raimondii, such as miR-156, miR-159, miR-160, miR-162, and miR-164 (Gong et al., 2013). Xue et al. (2013) confirmed the expression of eight fiber elongation-related and 257 novel microRNAs in elongating cotton fiber cells. Thus, miRNAs are becoming a novel target for improving plant yield, quality, and response to environmental stress (Zhang and Wang, 2015). In the present study, the qRT-PCR was carried out to study the miRNA expression patterns in four different tissues in the model cotton species, G. raimondii, which has not been done before and to further shed light on the specific roles of these miRNAs in cotton growth and development.

According to the qRT-PCR results, eight miRNAs, including miR-159, miR-162, miR-164, miR-172, miR-390, miR-395, miR-397, and miR-398, exhibited exclusively high expression levels in flower buds, suggesting that these miRNAs may play significant roles in floral development. Such expression patterns were consistent with several functional studies in Arabidopsis as well as some other plant species (Aukerman and Sakai, 2003; Chen, 2004; Lauter et al., 2005). It has been reported that miR 172 targets a class of APETALA2 (AP2)-like genes (e.g. $A P 2, T O E 1$, and TOE2) that encode transcription factors involved in plant flowering and floral organ identity (Aukerman and Sakai, 2003; Chen, 2004). According to previous studies, overexpressed miR-172 in Arabidopsis resulted in early flowering and floral organ identity defects, indicating that miR-172 plays a crucial role in the regulation of flower development as a translational repressor of AP2-like genes (Aukerman and Sakai, 2003; Chen, 2004). Lauter et al. (2005) also demonstrated that miR-172 contributed to the transition from vegetative growth to reproductive phase in maize by downregulating glossy 15 genes that function to keep the vegetative phase, which further supported the role of miR-172 in floral development. This suggests that miR-172 is a biomarker for phase change. Our study also shows that miR-172 was highly expressed in flower-related tissues. In addition, miR-159 was also shown to participate in the floral development (Achard et al., 2004). It has been confirmed that miR-159 targets at least two MYB transcription factor coding genes, namely MYB33 and MYB65, which act as important regulators of floral initiation and floral 
organ development, especially anther and pollen development (Gocal et al., 2001; Kaneko et al., 2004; Millar and Gubler, 2005). Phenotypic analyses of Arabidopsis, barley, and rice revealed that elevated levels of miR-159 caused a delay in flowering under short day photoperiods as a result of decreased MYB33 transcript levels, and perturbation of anther development leading to reduction in floral fertility, similar to myb33/myb65 double mutants (Achard et al., 2004; Millar and Gubler, 2005). Moreover, functional studies also revealed that miR-164 was involved in floral development (Laufs et al., 2004; Mallory et al., 2004). Previous studies indicated that miR-164 targeted five members (NACl, CUCl, CUC2, At5g07680, and At5g61430) of the NACdomain gene family that play significant roles in embryonic, vegetative, and floral development (Rhoades et al., 2002; Laufs et al., 2004; Mallory et al., 2004; Guo et al., 2005). Overexpression of miR-164 in wild type plants caused aberrant floral morphology such as one to four extra petals and fusion of floral organs (Laufs et al., 2004; Mallory et al., 2004). Additionally, miR-390 was reported to play a role in regulation of flowering timing (RubioSomoza and Weigel, 2011). miR-390 contributed to the delay of flowering onset by inhibiting the activities of transcription factors ARF3 and ARF4 which function as positive regulators of the transition from the juvenile to adult vegetative phase (Fahlgren et al., 2006; Garcia, 2008; Montgomery et al., 2008; RubioSomoza and Weigel, 2011; Endo et al., 2013). Our miRNA expression profile results also showed that the expression level of miR-164 was relatively high in shoots, besides flower buds, implying that the function of miR-164 is not only limited to floral development but it may also play an important role in shoot development. This hypothesis has been supported by several functional studies (Laufs et al., 2004; Mallory et al., 2004; Sieber et al., 2007). It was demonstrated that $\mathrm{CUC1}$ and $\mathrm{CUC2}$ are two important targets of miR-164 necessary for shoot meristem formation (Aida and Tasaka, 2006). Loss-of-function of miR-164 in wild type plants resulted in severe defects during shoot development (Laufs et al., 2004; Mallory et al., 2004; Sieber et al., 2007). Moreover, it is also remarkable that certain miRNAs such as miR-166 and miR-160 were extremely highly expressed in all the four tissues tested compared with other miRNAs investigated, suggesting that they may play regulatory roles at multiple development stages. Such expression profiles were in strong agreement with functional studies. miR-166, for instance, was not only reported to play crucial roles in the regulation of shoot apical meristem, but also reported to regulate leaf polarity, floral development as well as vascular development (Kim et al., 2005; Jung and Park, 2007; Xu et al., 2007; Zhu et al., 2011). It has been demonstrated that miR-166 targeted a subset of genes in the HD-ZIP transcription factor gene family, including CORONA (CNA)/ATHB15, ATHB8PHA BULOSA (PHB), REVOLUTA (REV), and PHAVO LUTA (PHV), which regulate a wide range of plant developmental processes, such as organ polarity, meristem initiation, leaf development, shoot meristem formation, floral development, and vascular development (McConnell et al., 2001; Otsuga et al., 2001; Emery et al., 2003; Ohashi-Ito and Fukuda, 2003; Bao et al., 2004; Bowman, 2004; Juarez et al., 2004; Zhong and Ye, 2004; Kim et al., 2005; Prigge et al., 2005). Jung and Park (2007) reported that elevated levels of miR-166 caused accelerated activity of shoot apical meristem and floral structure defects, while Kim et al. (2005) showed that overexpression of miR-166 in wild type plants could also lead to an altered plant vascular system by repressing the activity of $A T H B 15$, one of the targets of miR-166 that play a role in vascular development.

\section{Conclusions}

This present study investigated the tissuespecific expression profiles of sixteen conserved miRNAs in G. raimondii. The results showed that the expression levels of these miRNAs varied significantly from one to another in a tissue-dependent manner. Nine miRNAs exhibited exclusively high expression levels in flower buds, suggesting that they may play significant roles in floral development. In addition, certain miRNAs such as miR-166 and miR-160 were extremely highly expressed in all the four tissues tested compared with other miRNAs investigated, suggesting that they may play regulatory roles at multiple development stages. This study will contribute to future studies on the functional characterizations of miRNAs in cotton. 


\section{Compliance with ethics guidelines}

Jun MA, Teng-long GUO, Qing-lian WANG, Kun-bo WANG, Run-run SUN, and Bao-hong ZHANG declare that they have no conflict of interest.

This article does not contain any studies with human or animal subjects performed by any of the authors.

\section{References}

Achard, P., Herr, A., Baulcombe, D.C., et al., 2004. Modulation of floral development by a gibberellinregulated microRNA. Development, 131(14):3357-3365. [doi:10.1242/dev.01206]

Aida, M., Tasaka, M., 2006. Genetic control of shoot organ boundaries. Curr. Opin. Plant Biol., 9(1):72-77. [doi:10. 1016/j.pbi.2005.11.011]

Aukerman, M.J., Sakai, H., 2003. Regulation of flowering time and floral organ identity by a microRNA and its APETALA2-like target genes. Plant Cell, 15(11):27302741. [doi:10.1105/tpc.016238]

Bao, N., Lye, K.W., Barton, M.K., 2004. MicroRNA binding sites in Arabidopsis class III HD-ZIP mRNAs are required for methylation of the template chromosome. Dev. Cell, 7(5):653-662. [doi:10.1016/j.devcel.2004.10. 003]

Bartel, D.P., 2004. MicroRNAs: genomics, biogenesis, mechanism, and function. Cell, 116(2):281-297. [doi:10. 1016/S0092-8674(04)00045-5]

Bowman, J.L., 2004. Class III HD-Zip gene regulation, the golden fleece of ARGONAUTE activity? Bioessays, 26(9):938-942. [doi:10.1002/bies.20103]

Carrington, J.C., Ambros, V., 2003. Role of microRNAs in plant and animal development. Science, 301(5631):336338. [doi:10.1126/science.1085242]

Chen, X.M., 2004. A microRNA as a translational repressor of APETALA2 in Arabidopsis flower development. Science, 303(5666):2022-2025. [doi:10.1126/science.1088060]

Emery, J.F., Floyd, S.K., Alvarez, J., et al., 2003. Radial patterning of Arabidopsis shoots by class III HD-ZIP and KANADI genes. Curr. Biol., 13(20):1768-1774. [doi:10. 1016/j.cub.2003.09.035]

Endo, Y., Iwakawa, H.O., Tomari, Y., 2013. Arabidopsis ARGONAUTE7 selects miR390 through multiple checkpoints during RISC assembly. EMBO Rep., 14(7): 652-658. [doi:10.1038/embor.2013.73]

Fahlgren, N., Montgomery, T.A., Howell, M.D., et al., 2006. Regulation of AUXIN RESPONSE FACTOR3 by TAS3 ta-siRNA affects developmental timing and patterning in Arabidopsis. Curr. Biol., 16(9):939-944. [doi:10.1016/j. cub.2006.03.065]

Garcia, D., 2008. A miRacle in plant development: role of microRNAs in cell differentiation and patterning. Semin. Cell Dev. Biol., 19(6):586-595. [doi:10.1016/j.semcdb. 2008.07.013]

Gocal, G.F., Sheldon, C.C., Gubler, F., et al., 2001. GAMYB-like genes, flowering, and gibberellin signaling in Arabidopsis. Plant Physiol., 127(4):1682-1693. [doi:

\subsection{4/pp.010442]}

Gong, L., Kakrana, A., Arikit, S., et al., 2013. Composition and expression of conserved microRNA genes in diploid cotton (Gossypium) species. Genome Biol. Evol., 5(12): 2449-2459. [doi:10.1093/gbe/evt196]

Guo, H.S., Xie, Q., Fei, J.F., et al., 2005. MicroRNA directs mRNA cleavage of the transcription factor $N A C 1$ to downregulate auxin signals for Arabidopsis lateral root development. Plant Cell, 17(5):1376-1386. [doi:10.1105/ tpc.105.030841]

Juarez, M.T., Kui, J.S., Thomas, J., et al., 2004. MicroRNAmediated repression of rolled leaf1 specifies maize leaf polarity. Nature, 428(6978):84-88. [doi:10.1038/nature 02363]

Jung, J.H., Park, C.M., 2007. miR166/165 genes exhibit dynamic expression patterns in regulating shoot apical meristem and floral development in Arabidopsis. Planta, 225(6):1327-1338. [doi:10.1007/s00425-006-0439-1]

Kaneko, M., Inukai, Y., Ueguchi-Tanaka, M., et al., 2004. Loss-of-function mutations of the rice GAMYB gene impair $\alpha$-amylase expression in aleurone and flower development. Plant Cell, 16(1):33-44. [doi:10.1105/tpc. 017327]

Kim, J., Jung, J.H., Reyes, J.L., et al., 2005. MicroRNAdirected cleavage of $A T H B 15$ mRNA regulates vascular development in Arabidopsis inflorescence stems. Plant J., 42(1):84-94. [doi:10.1111/j.1365-313X.2005.02354.x]

Kurihara, Y., Watanabe, Y., 2004. Arabidopsis micro-RNA biogenesis through Dicer-like 1 protein functions. PNAS, 101(34):12753-12758. [doi:10.1073/pnas.0403115101]

Laufs, P., Peaucelle, A., Morin, H., et al., 2004. MicroRNA regulation of the $C U C$ genes is required for boundary size control in Arabidopsis meristems. Development, 131(17): 4311-4322. [doi:10.1242/dev.01320]

Lauter, N., Kampani, A., Carlson, S., et al., 2005. MicroRNA172 down-regulates glossy15 to promote vegetative phase change in maize. $P N A S$, 102(26): 9412-9417. [doi:10.1073/pnas.0503927102]

Lee, Y., Kim, M., Han, J., et al., 2004. MicroRNA genes are transcribed by RNA polymerase II. EMBO J., 23(20): 4051-4060. [doi:10.1038/sj.emboj.7600385]

Liu, N., Tu, L., Tang, W., et al., 2014. Small RNA and degradome profiling reveals a role for miRNAs and their targets in the developing fibers of Gossypium barbadense. Plant J., 80(2):331-344 [doi:10.1111/tpj. 12636]

Ma, J., Wang, Q., Sun, R., et al., 2014. Genome-wide identification and expression analysis of TCP transcription factors in Gossypium raimondii. Sci. Rep., 4:6645. [doi:10.1038/srep06645]

Mallory, A.C., Dugas, D.V., Bartel, D.P., et al., 2004. MicroRNA regulation of NAC-domain targets is required for proper formation and separation of adjacent embryonic, vegetative, and floral organs. Curr. Biol., 14(12):1035-1046. [doi:10.1016/j.cub.2004.06.022]

McConnell, J.R., Emery, J., Eshed, Y., et al., 2001. Role of PHABULOSA and PHAVOLUTA in determining radial 
patterning in shoots. Nature, 411(6838):709-713. [doi:10. 1038/35079635]

Millar, A.A., Gubler, F., 2005. The Arabidopsis GAMYB-like genes, MYB33 and MYB65, are microRNA-regulated genes that redundantly facilitate anther development. Plant Cell, 17(3):705-721. [doi:10.1105/tpc.104.027920]

Millar, A.A., Waterhouse, P.M., 2005. Plant and animal microRNAs: similarities and differences. Funct. Integr. Genomics, 5(3):129-135. [doi:10.1007/s10142-005-0145-2]

Montgomery, T.A., Howell, M.D., Cuperus, J.T., et al., 2008. Specificity of ARGONAUTE7-miR390 interaction and dual functionality in TAS3 trans-acting siRNA formation. Cell, 133(1):128-141. [doi:10.1016/j.cell.2008.02.033]

Ohashi-Ito, K., Fukuda, H., 2003. HD-Zip III homeobox genes that include a novel member, ZeHB-13 (Zinnia)/ATHB-15 (Arabidopsis), are involved in procambium and xylem cell differentiation. Plant Cell Physiol., 44(12):13501358. [doi:10.1093/pcp/pcg164]

Otsuga, D., Deguzman, B., Prigge, M.J., et al., 2001. REVOLUTA regulates meristem initiation at lateral positions. Plant J., 25(2):223-236. [doi:10.1046/j.1365313x.2001.00959.x]

Papp, I., Mette, M.F., Aufsatz, W., et al., 2003. Evidence for nuclear processing of plant micro RNA and short interfering RNA precursors. Plant Physiol., 132(3):13821390. [doi:10.1104/pp.103.021980]

Park, M.Y., Wu, G., Gonzalez-Sulser, A., et al., 2005. Nuclear processing and export of microRNAs in Arabidopsis. PNAS, 102(10):3691-3696. [doi:10.1073/pnas.0405570102]

Prigge, M.J., Otsuga, D., Alonso, J.M., et al., 2005. Class III homeodomain-leucine zipper gene family members have overlapping, antagonistic, and distinct roles in Arabidopsis development. Plant Cell, 17(1):61-76. [doi:10.1105/tpc.104.026161]

Rhoades, M.W., Reinhart, B.J., Lim, L.P., et al., 2002. Prediction of plant microRNA targets. Cell, 110(4):513520. [doi:10.1016/S0092-8674(02)00863-2]

Rubio-Somoza, I., Weigel, D., 2011. MicroRNA networks and developmental plasticity in plants. Trends Plant Sci., 16(5):258-264. [doi:10.1016/j.tplants.2011.03.001]

Sieber, P., Wellmer, F., Gheyselinck, J., et al., 2007. Redundancy and specialization among plant microRNAs: role of the MIR164 family in developmental robustness. Development, 134(6):1051-1060. [doi:10.1242/dev.02817]

Xie, F.L., Jones, D.C., Wang, Q.L., et al., 2015. Small RNA sequencing identifies miRNA roles in ovule and fiber development. Plant Biotechnol. J., 13(3):338-352. [doi: 10.1111/pbi.12296]

Xu, L., Yang, L., Huang, H., 2007. Transcriptional, posttranscriptional and post-translational regulations of gene expression during leaf polarity formation. Cell Res.,
17(6):512-519. [doi:10.1038/cr.2007.45]

Xue, W., Wang, Z., Du, M., et al., 2013. Genome-wide analysis of small RNAs reveals eight fiber elongationrelated and 257 novel microRNAs in elongating cotton fiber cells. BMC Genomics, 14:629. [doi:10.1186/14712164-14-629]

Zhang, B.H., 2015. MicroRNA: a new target for improving plant tolerance to abiotic stress. J. Exp. Bot., 66(7): 1749-1761. [doi:10.1093/jxb/erv013]

Zhang, B.H., Wang, Q.L., 2015. MicroRNA-based biotechnology for plant improvement. J. Cell. Physiol., 230(1):1-15. [doi:10.1002/jcp.24685]

Zhang, B.H., Pan, X.P., Cobb, G.P., et al., 2006. Plant microRNA: a small regulatory molecule with big impact. Dev. Biol., 289(1):3-16. [doi:10.1016/j.ydbio.2005.10.036]

Zhang, B.H., Wang, Q.L., Pan, X.P., 2007. MicroRNAs and their regulatory roles in animals and plants. J. Cell. Physiol., 210(2):279-289. [doi:10.1002/jcp.20869]

Zhong, R., Ye, Z.H., 2004. amphivasal vascular bundle 1, a gain-of-function mutation of the IFL1/REV gene, is associated with alterations in the polarity of leaves, stems and carpels. Plant Cell Physiol., 45(4):369-385. [doi:10. 1093/pcp/pch051]

Zhu, H., Hu, F., Wang, R., et al., 2011. Arabidopsis Argonaute10 specifically sequesters miR166/165 to regulate shoot apical meristem development. Cell, 145(2):242-256. [doi:10.1016/j.cell.2011.03.024]

\section{中文概要}

题 目: MicroRNA 在雷蒙德氏棉中的表达

目 的: 探索 16 个保守 microRNA 在雷蒙德氏棉中的表 达情况。

创新点: 首次研究了 microRNA 在雷蒙德氏棉四个组织的 表达情况。

方 法: 设计 16 个 microRNA 的引物, 并提取雷蒙德氏 棉四个不同组织的 RNA 进行实时荧光定量聚合 酶链式反应（qRT-PCR）。

结 论: 在不同的组织中, 这些 microRNA 的表达水平差 异很大。包括 miR-159、miR-162、miR-164、 miR-172、miR-390、miR-395、miR-397 和 miR-398 在内的 8 个 microRNA 在花蕾中表达含量非常 高, 而另外一些 microRNA, 例如 miR-166 和 miR-160, 在四个不同组织中都有很高的表达量。

关键词: 棉花; microRNA; 表达谱; 实时苂光定量聚合 酶链式反应（qRT-PCR）; 雷蒙德氏棉 